\title{
Author correction: Nanoparticle-mediated local depletion of tumour-associated platelets disrupts vascular barriers and augments drug accumulation in tumours
}

Suping Li, Yinlong Zhang, Jing Wang, Ying Zhao, Tianjiao Ji, Xiao Zhao, Yanping Ding, Xiaozheng Zhao, Ruifang Zhao, Feng Li, Xiao Yang, Shaoli Liu, Zhaofei Liu, Jianhao Lai, Andrew K. Whittaker, Gregory J. Anderson, Jingyan Wei and Guangjun Nie

Nature Biomedical Engineering https://doi.org/10.1038/s41551-017-0115-8 (2017); published online 24 July 2017; corrected online 2 August 2017.

In the version of the Supplementary Information originally published, in Supplementary Fig. 8a, in the bottom row, the left-most image ('Control') was not the correct image; this has now been replaced. 